\title{
Liver resection for hepatocellular carcinoma in patients with hematological malignancies
}

\author{
Hui-Chen Lin', Yang-Shen Yang ${ }^{2}$, Chieh-Lin Jerry Teng ${ }^{2}$, Ching-Hui Shen ${ }^{3}$, Yee-Gee Jan ${ }^{4}$, Shao-Bin Cheng ${ }^{1,7}$, \\ Cheng-Chung $\mathrm{Wu}^{1,5,6,7^{*}}$, Yi-Ling Lin' ${ }^{1}$ Chu-Chun Huang ${ }^{1}$ and Fang-Ku P'eng ${ }^{1,5}$
}

\begin{abstract}
Background: Improvements in antimetabolite drugs have prolonged the survival of patient with hematological malignancies. However, these drugs may have hepatotoxic side effects and may induce acute liver failure, chronic liver fibrosis, cirrhosis, or even hepatocellular carcinoma (HCC). Although liver resection remains a curative option for HCC, its role in HCC with hematological malignancies has never been fully explored.

Methods: A retrospective review of 1725 patients who underwent curative liver resection for newly diagnosed HCC between 1994 and 2016 was conducted. Among these patients, 16 had a history of hematological malignancies (HM group). Their hematological malignancies were well-controlled at the time of liver resection. The clinicopathological characteristics of the HM group, along with their short- and long-term outcomes after liver resection, were compared with those of the other 1709 patients without hematological malignancy (non-HM group).

Results: All HM group patients were seropositive for hepatitis marker surface for hepatitis B and C. No significant differences were observed in any background characteristics between the two groups. The postoperative complication rate and 90-day mortality in the HM and non-HM groups were 25 and 20.4\%, $P=0.754$, and 0 and $0.6 \%, P=1.000$, respectively. The 5-year disease-free and overall survival rates for the HM and non-HM groups were 42.3 and $35.1 \%, P=0.552$, and 69.5 and $56.9 \%, P=0.192$, respectively.

Conclusions: Hepatitis markers should be examined during chemotherapy for hematological malignancies. Regular liver imaging studies are recommended for seropositive cases. When HCC occurs secondary to a well-controlled hematological malignancy, liver resection is suggested in selected patients.
\end{abstract}

Keywords: Hepatocellular carcinoma, Liver resection, Hematological malignancy

\section{Background}

Outcomes of hematological malignancies have improved substantially and survival times have markedly increased due to the progression of antimetabolite therapies [1]. However, the hepatotoxic side effects of antimetabolite drugs are an important consideration [2-5]. Acute hepatocyte necrosis and fulminant liver failure may occur after administration of these agents, especially in patients with chronic hepatitis $\mathrm{B}$ or $\mathrm{C}$ infection $[2,3]$.

\footnotetext{
* Correspondence: he@vghtc.gov.tw

1 Department of Surgery, Taichung Veterans General Hospital, Section, 4, No. 1650, Taiwan Boulevard, Taichung, Taiwan

${ }^{5}$ Department of Surgery, School of Medicine, National Yang-Ming University, Taipei, Taiwan

Full list of author information is available at the end of the article
}

Moreover, antimetabolite drugs may cause methylation of mitochondrial DNA in hepatocytes, resulting in phenotypic changes, and may induce malignant change in hepatocytes leading to subsequent primary hepatocellular carcinoma (HCC) formation $[4,5]$. Therefore, liver fibrosis, cirrhosis, and $\mathrm{HCC}$ have been reported after long-term use of these drugs [4]. Moreover, as Taiwan is an endemic area of hepatitis B, HCC has consistently ranked as the first or second leading cause of cancer death in the past 20 years. Most HCC patients in Taiwan are seropositive for hepatitis B surface antigen (HBsAg) and/or anti-hepatitis $\mathrm{C}$ antibody (anti-HCV) [6-16]. When patients with hepatitis also have hematological malignancies, the hepatocytes that have been injured by hepatitis $\mathrm{B}$ or $\mathrm{C}$ may be more susceptible to 
antimetabolite-induced insults and accelerated HCC formation [4-8]. We have observed the development of $\mathrm{HCC}$ in patients with hematological malignancies.

At present, radiofrequency ablation (RFA), liver resection, and liver transplantation are well-recognized as curative options for HCC [6, 7]. However, these strategies have their advantages and limitations. Appropriate case selection with multimodality therapies may provide long-term survival and optimal quality of life [6-8]. Among these strategies, liver resection remains the most widely adopted classical curative modality for HCC [9-18]. However, liver resection is a highly complex procedure with a high complication rate despite its low mortality in high-volume centers [9].

To the best of our knowledge, the role of liver resection for HCC in patients with hematological malignancies has never been investigated. In this study, a retrospective analysis of prospectively collected data from HCC patients who underwent liver resection was conducted. The aim of the study was to elucidate the benefits of liver resection for $\mathrm{HCC}$ in this patient cohort.

\section{Methods}

This study was approved by the institutional review board (IRB). A retrospective review of prospectively collected data from 1725 consecutive patients who underwent curative liver resection for newly diagnosed HCC between 1994 and 2016 was conducted. Curative resection was defined as the removal of tumors that were preoperatively detected by imaging studies or the removal of tumors that were detected intraoperatively by palpation or ultrasonography [10-18].

Among the HCC patients, $16(0.9 \%)$ had a history of varied hematological malignancies. All patients received generally recommended regimens of systemic chemotherapies [1]. The HCCs in 3 patients were detected during the course of chemotherapy, while the HCCs in the other 13 patients were detected at an average of 79 (range, 9-174) months after hematological treatment. The hematological malignancies included large B-cell lymphoma in eight patients (nodal type 5 , extranodal type 3 (stomach, 1 , orbit floor 1 , colon 1 ), multiple myeloma in three, chronic myelocytic leukemia in two patients, chronic lymphocytic leukemia in two patients, and acute myelocytic leukemia in one patient. For the three HCCs (one lymphoma, multiple myeloma, and chronic lymphocytic leukemia) that were found during the course of chemotherapy, the chemotherapy (synchronous HCC) was temporarily discontinued for 4 weeks and was then restarted 2 weeks after total recovery from the hepatectomy or after complete postoperative healing had occurred. The policies and protocols of the systemic anticancer therapies for these hematological malignancies are listed in Table 1. Two patients had $\geq 3$ malignancies (lymphoma, papillary thyroid carcinoma, stomach cancer and HCC in 1 patient; multiple myeloma, basal cell carcinoma of the chest wall, and $\mathrm{HCC}$ in 1 patient).
Table 1 Anticancer drugs for hematological malignancies

\begin{tabular}{ll}
\hline Hematologic malignancy & Anticancer drugs \\
\hline Son-Hodgkin's lymphoma & \\
Aggressive lymphoma & Modest chemotherapy \\
Diffuse large B cell lymphoma & Intensive chemotherapy \\
& Standard first-line chemotherapy \\
& Rituximab plus CHOP \\
& (cyclophosphamide, vincristine, \\
& doxorubicin, prednisolone) \\
& 1st-line thalidomide; ASCT \\
Multiple myeloma & (autologous stem cell transplant) \\
& 2nd-line bortezomib (Velcade), \\
& thalidomide, dexamethasone \\
& Lenalidomide \\
& CVP (cyclophosphamide, \\
Chronic lymphocytic leukemia & vincristine, prednisolone) \\
& Bendamustine, rituximab, \\
& fludarabine \\
Cyte myeloid leukemia & Cytosine arabinoside \\
& Idarubicin
\end{tabular}

\section{Patient selection criteria for $\mathrm{HCC}$ resection}

\section{Assessments of HCC patients who underwent liver resection} The hematological malignancies of all patients were considered to be well-controlled by hematologists (YSY and JLT). The extrahepatic malignancies in two patients who had $\geq 3$ primary malignancies were also considered to be controlled at the time of HCC diagnosis. The results of conventional liver function tests, seropositivity for $\mathrm{HBsAg}$ and anti-HCV, and the serum $\alpha$-fetoprotein (AFP) level were determined [9-15]. The indocyanine-green 15-min retention rate (ICG R15) was determined for each patient. Child-Pugh grading for cirrhotic liver function [16] was conducted for all patients. If the patient's general condition fulfilled the criteria for American Society of Anesthesiology (ASA) class 1 or 2, liver resection was indicated [9]. Computed tomography $(\mathrm{CT})$ or magnetic resonance imaging (MRI) of the liver was performed in all patients. The extent of liver resection was based on the ICG R15, and tumor extension was estimated using a modified version of the Makuuchi criteria for cirrhotic liver resection [9, 11-15]. Concomitant splenectomy was performed if the patient had hypersplenic thrombocytopenia (platelet count $<8 \times 10^{4} / \mathrm{mm}^{3}$ ) [13]. Preoperative gastroduodenal endoscopy was performed in all patients. If severe gastro-esophageal varices were found, they were treated by endoscopic sclerotherapy or variceal ligation [14]. Perioperative heparin-free hemodialysis was performed for patients with end-stage renal disease (from 1 day before surgery to postoperative day 7) [15].

During surgery, intraoperative ultrasonography was routinely performed to define the location of major intrahepatic vessels and tumors that were not visible or impalpable. The liver parenchyma was transected using the Kelly clamp 
crush technique with intermittent hepatic inflow blood occlusion under low central venous pressure (approximately $5 \mathrm{~cm} \mathrm{H}_{2} \mathrm{O}$ ), which was maintained by a senior anesthesiologists. A limited blood transfusion policy was applied to all patients.

The resected specimens were sent to a pathologist (YGJ) to determine the pathologic characteristics of HCC. UICC-TNM tumor staging (8th edition) [17] was applied to each patient for tumor staging after pathological examination. Cirrhosis severity was assessed using the Ishak scoring system [18].

Operative mortality was defined as a death within 90 days postoperatively. The Clavien-Dindo complication severity [19] was also evaluated in patients with complications. After undergoing hepatectomy, patients were followed up at both surgery and hematology out-patient clinics. If HCC recurred, patients were treated by re-resection, radiofrequency ablation (after 2000, for tumor with $\leq 3$ nodules, each $\leq$ $3 \mathrm{~cm}$ ), transarterial chemoembolization (TACE) [19], Sorafenib (after 2009 in our hospital) or conservative treatment as appropriate.

\section{Statistical analysis}

The clinicopathological characteristics of the 16 patients with a history of hematologic malignancies (HM group) were compared with those of the other 1709 patients without hematologic malignancies (non-HM group). The early postoperative results and the long-term disease-free (DFS) and overall survival (OS) rates were also compared between the two groups.

Continuous variables are expressed as medians (ranges) and were compared using the Mann-Whitney $U$ test. Frequencies were compared using Fisher's exact test or Pearson's $\chi^{2}$ test as appropriate. All patients were followed up until June 2017. The Kaplan-Meier life table method was used to calculate the DFS and OS rates. The log-rank test was used to compare the difference in survival between the two groups.

\section{Results}

The clinical characteristics of all patients who underwent liver resection are shown in Table 2 [20]. No significant differences were observed in any of the variables between the two groups. Notably, all patients in the HM group

Table 2 Background characteristics of HCC patients who underwent hepatectomy

\begin{tabular}{|c|c|c|c|}
\hline Characteristics & $\begin{array}{l}\text { HM group } \\
(n=16)\end{array}$ & $\begin{array}{l}\text { Non-HM group } \\
(n=1709)\end{array}$ & $P$ value \\
\hline Sex (male:female) & 11 (68.8\%):5 (31.2\%) & 1009 (59.0\%):700 (41.0\%) & 0.595 \\
\hline Age & $64(32-80)$ & $61.5(18-95)$ & 0.877 \\
\hline Hepatitis status & & & 0.815 \\
\hline $\mathrm{B}+\mathrm{C}+$ & $2(12.5 \%)$ & $82(4.0 \%)$ & \\
\hline $\mathrm{B}+\mathrm{C}-$ & $8(50.0 \%)$ & $784(46.1 \%)$ & \\
\hline $\mathrm{B}-\mathrm{C}+$ & $6(37.5 \%)$ & $579(34.1 \%)$ & \\
\hline $\mathrm{B}-\mathrm{C}-$ & $0(0 \%)$ & $264(14.9 \%)$ & \\
\hline $\operatorname{AFP}(\mathrm{ng} / \mathrm{ml})$ & $18.4(5.19-189)$ & $27.2(19-10,906)$ & 0.097 \\
\hline Cirrhosis & $14(87.5 \%)$ & $1271(75.2 \%)$ & 0.384 \\
\hline ICG R15 (\%) & $16.4(8.9-271)$ & $13.6(5.1-50.1)$ & 0.472 \\
\hline Splenectomy & $2(12.5 \%)$ & $130(7.7 \%)$ & 0.231 \\
\hline Association with GEV & $1(6.7 \%)$ & $211(12.4 \%)$ & 0.375 \\
\hline \multicolumn{4}{|c|}{ Comorbidities (other than HCC and hematologic malignancies) } \\
\hline Heart disease & 1 & 114 & 1.0 \\
\hline Respiratory disease & 0 & 54 & 1.0 \\
\hline Uremia & 0 & 54 & 1.0 \\
\hline Old stroke & 1 & 47 & 0.365 \\
\hline AIDS & 0 & 5 & 1.0 \\
\hline Malnutrition & 0 & 4 & 1.0 \\
\hline Heavy-metal intoxication & 0 & 2 & 1.0 \\
\hline Alcohol overintake $^{a}$ & 1 & 102 & 1.0 \\
\hline Lupus erythematosus & 1 & 11 & 0.106 \\
\hline
\end{tabular}

$B+C+$ seropositive for $\mathrm{HBsAg}$ and AntiHCV; $B+C$ - seropositive for $\mathrm{HBsAg}$, seronegative for AntiHCV; $B-C+$ seronegative for $\mathrm{HBs} A g$, seropositive for AntiHCV; $B-C$ - seronegative for HBsAg and AntiHCV; ICG R15 ICG 15-min retention rate; GEV gastroesophageal varices

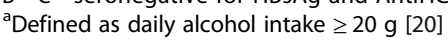


were seropositive for $\mathrm{HBsAg}$ and/or anti-HCV, although the data were not significantly different from those of patients in the non-HM group.

Early post-operative results are shown in Table 3. No significant differences were found for any complications or severities (Clavien-Dindo classification ${ }^{17}$ ) between the two groups. The 90-day incidence of mortality after surgery in the HM and non-HM groups were 0 and 11 $(0.6 \%)(P=1.000)$, respectively.

The pathological characteristics of the resected specimens are listed in Table 4. No significant differences were found between the two groups.

Repeat hepatectomy was performed on two patients in the HM group and on 298 patients in the non-HM group. As shown in Fig. 1, the 2-, 5-, and 10-year-DFS rates for HCC in the HM group were 66.7, 42.3, and $31.7 \%$, while the 2-, 5-, and 10-year -DFS rates for HCC in the non-HM group were $57.2,35.1$, and $21.1 \%$, respectively $(P=0.552)$. Figure 2 shows that the 2-, 5-, and 10-year-OS rates after resection of HCC in the HM group were 79.4, 69.5 , and $69.5 \%$, while the 2-, 5-, and 10-year-OS rates for $\mathrm{HCC}$ in the non-HM group were $78.4,56.9$, and $34.1 \%$, respectively $(P=0.197)$. No patients in the HM group had a recurrence of or died from a hematological malignancy. One 53-year-old HBsAg-positive woman underwent S8 liver resection of a 6-cm HCC (AJCC-TNM stage T2N0M0) on February 12, 2006. She underwent total

Table 3 Early postoperative result after hepatectomy

\begin{tabular}{|c|c|c|c|}
\hline & $\begin{array}{l}\text { HM group } \\
(n=16)\end{array}$ & $\begin{array}{l}\text { Non-HM group } \\
(n=1709)\end{array}$ & $P$ value \\
\hline Total Pringle clamping (min) & $45.0(23.6-84.6)$ & $59.6(0-168.8)$ & 0.112 \\
\hline Operation time (h) & $4.58(3.85-5.42)$ & $4.17(3.5-11.14)$ & 0.328 \\
\hline Op blood loss (ml) & $625(340-7500)$ & $500(50-8500)$ & 0.545 \\
\hline No blood transfusion & $13(81.3 \%)$ & $1467(86.0 \%)$ & 0.482 \\
\hline Postop stay (day) & $9(7-29)$ & $8(7-48)$ & 0.774 \\
\hline Complication & $4(25.0 \%)$ & $341(20.4 \%)$ & 0.754 \\
\hline Bile leakage & 2 & 160 & \\
\hline Abdominal abscess & 1 & 104 & \\
\hline Ascites & 1 & 94 & \\
\hline Wound infection & 0 & 57 & \\
\hline Postop bleeding & 0 & 31 & \\
\hline Prolonged jaundice & 0 & 40 & \\
\hline $\mathrm{Ml}$ & 0 & 11 & \\
\hline Stroke & 0 & 4 & \\
\hline Clavien-Dindo grading & & & 0.788 \\
\hline Grade 3 & 1 & 82 & \\
\hline Grade 4 & 1 & 29 & \\
\hline Grade 5 & 0 & 11 & \\
\hline 90-day mortality & $0(0.0 \%)$ & $11(0.6 \%)$ & 1.000 \\
\hline
\end{tabular}

Op operative, Postop postoperative
Table 4 Pathological characteristics of the resected HCC

\begin{tabular}{|c|c|c|c|}
\hline & $\begin{array}{l}\text { HM group } \\
(n=16)\end{array}$ & $\begin{array}{l}\text { Non-HM group } \\
(n=1709)\end{array}$ & $P$ value \\
\hline Extent of liver resection & & & 0.925 \\
\hline$\leq 1$ segment* & $10(62.5 \%)$ & $1031(61.5 \%)$ & \\
\hline $1.1-2.9$ segments* & $4(25.0 \%)$ & $403(24.0 \%)$ & \\
\hline$\geq 3$ segments* & $2(12.5 \%)$ & $242(14.1 \%)$ & \\
\hline Resected liver weights (g) & $135(48.8-1200)$ & $105(50-1600)$ & 0.818 \\
\hline Tumor size $(\mathrm{cm})$ & $4.6(2.6-8.0)$ & $5.2(3-17)$ & 0.921 \\
\hline Tumor number $\geq 2$ & $3(18.8 \%)$ & $317(18.9 \%)$ & 0.999 \\
\hline Presence of vascular invasion & $8(50 \%)$ & 994 (58.2\%) & 0.918 \\
\hline Presence of satellite nodules & $6(37.5 \%)$ & $772(45.2 \%)$ & 0.890 \\
\hline Presence of capsule & $9(56.3 \%)$ & $899(52.8 \%)$ & 0.982 \\
\hline TNM stage $^{\#}$ & & & 0.815 \\
\hline l & $8(50 \%)$ & $687(39.0 \%)$ & \\
\hline$\|$ & $4(25 \%)$ & $515(30.6 \%)$ & \\
\hline III & $4(25 \%)$ & 493 (29.2\%) & \\
\hline IV & $0(00 \%)$ & $20(1.2 \%)$ & \\
\hline Tumor differentiation & & & 0.903 \\
\hline WD & 2 & 197 & \\
\hline$M D+P D$ & 14 & 502 & \\
\hline Resection margin $(\mathrm{mm})$ & $5(1.5-14.0)$ & $3(0-19.0)$ & \\
\hline
\end{tabular}

${ }^{*}$ Couinaud's Segment ${ }^{\# 7} 7$ th edition (2009)

thyroidectomy for papillary carcinoma of the thyroid in 1990. She also underwent a D2 distal gastrectomy for gastric carcinoma in 1993 (AJCC-TNM stage T4aNOM0). She had a malignant lymphoma (B-cell type) in a neck lymph node and underwent chemotherapy in 1995. An S6 recurrent HCC was resected on December 16, 2011. Neck lymphatic recurrence of thyroid cancer was diagnosed in July 2013, and a modified neck dissection with radio-

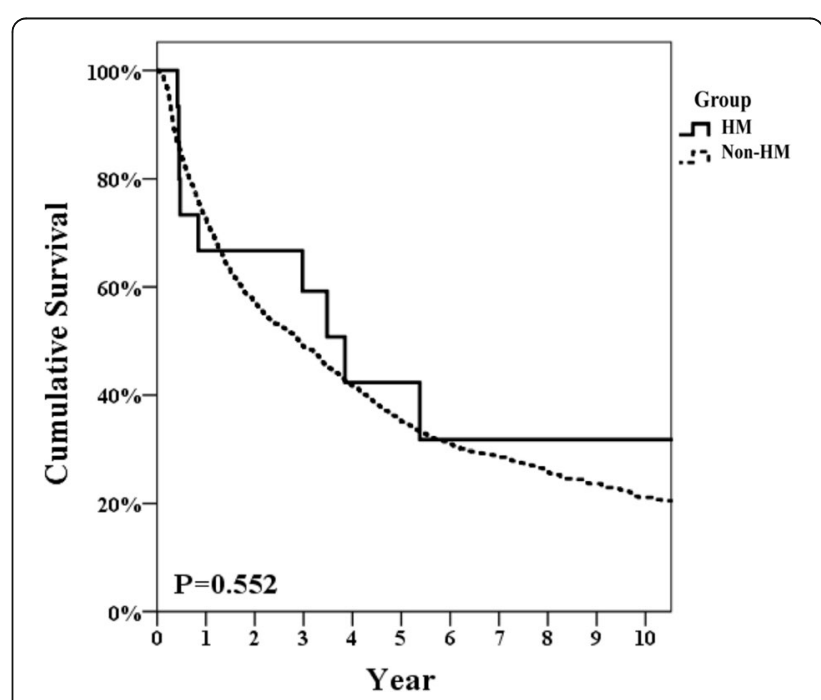

Fig. 1 Disease-free survival rate of HCC patients after resection 


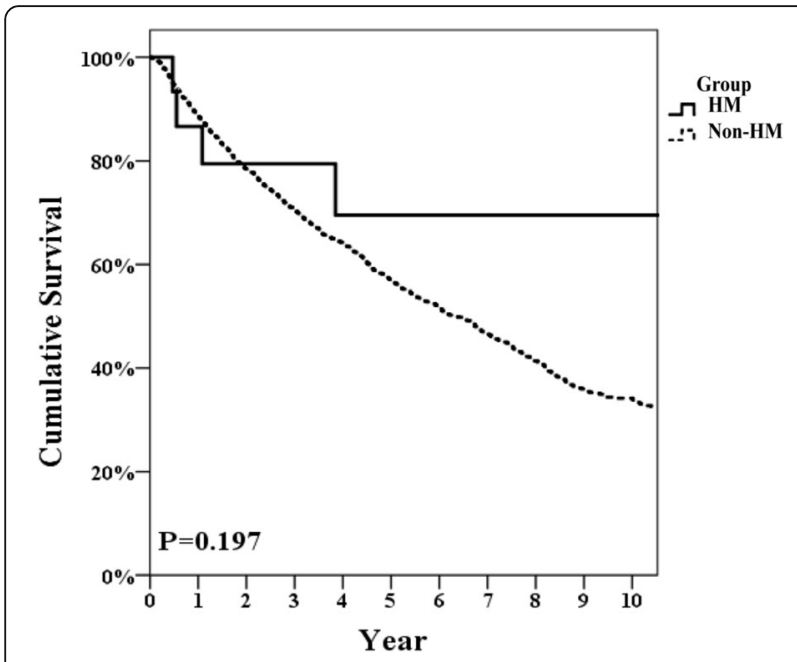

Fig. 2 Overall survival rate of HCC patients after resection

iodine ablation was performed. She remains disease-free at the time of this report, 11 years after the first hepatectomy and 5.5 years after the second hepatectomy.

\section{Discussion}

In this study, HCC patients with hematological malignancies (HM group) were categorized as having a multiple primary malignancy (MPM) disease. The term MPM was first proposed by Billroth in 1884 [21]. In 1932, Warren and Gates conducted a literature review that included autopsy cases and found evidence of more than 1000 cases of MPM [22]. With recent improvements in diagnostic tools and prolonged survival rates after malignancy treatment, the incidence of MPM has gradually increased. Berrington de Gonzalez, et al. [23] reported that 18\% of cancer patients who had received radiotherapy in the USA had MPM. In the European Union, the incidence of MPM was reported to be $11 \%$ [24]. For MPM that included HCC, a study conducted in Korea in 2012 reported an incidence rate of $11.5 \%$ [25].

The cause of secondary liver malignancy formation in patients with hematologic malignancy is not wellrecognized. In our series, not surprisingly, hepatitis $B$ and $\mathrm{C}$ still played important roles in HCC formation in HM group patients, as all patients were seropositive for HBsAg and/or anti-HCV. A possible mechanism involves hepatocyte injury (either acute or chronic) by antimetabolite drugs, which can cause genetic changes and cancer cell formation. All hematologic malignancies developed in patients whose conditions were wellcontrolled. Taiwan is an endemic area for hepatitis B virus; a large proportion of the general population is seropositive for HBsAg. When affected individuals receive antimetabolite drugs, the hepatotoxic side effects of these drugs may cause liver cell damage or liver cell carcinogenesis by methylation of mitochondria [4-7]. In the past, physicians in our institution reported the use of large doses of antimetabolite drugs during pulse therapy for active collagen disease with massive liver necrosis requiring liver transplantation [2]. Thereafter, they established a guideline to perform routine evaluations of liver function and serum HBsAg and anti-HCV seropositivity for the early detection of liver injury after chemotherapy [3].

In this series, all HM group patients were seropositive for hepatitis markers. As with general HCC populations who have a high incidence of positive serum hepatitis markers, regular liver imaging studies should be mandatory in HBsAg- or anti-HCV-positive patients undergoing chemotherapy for hematologic malignancies to determine the presence of liver tumors. Moreover, HCC formation and hepatotoxic conditions such as acquired immunodeficiency syndrome (AIDS), malnutrition, alcohol overintake (defined as daily alcohol intake $\geq 20 \mathrm{~g}$ [20]), and heavy metal toxicity can be researched using present study model. Nevertheless, the incidence of these conditions in our HCC patients are very low (Table 2). Yet, the true mechanism of $\mathrm{HCC}$ formation in these circumstances remained unknown.

The outcome of MPM patients remains controversial. Long-term survival could be achieved in patients whose malignancies are well-controlled. The prognosis of unresectable HCC in patients with hematological malignancy is usually dismal [4]. The association of HCC and hematological malignancy is unique; the incidence was only $0.93 \%$ in our series over 22 years. A report from Beijing, China, in which a 26-year follow-up study was conducted, reported that the most common secondary cancers with HCC were lung cancer, colorectal cancer, and thyroid cancer. Only 1 of $40 \mathrm{HCC}$ patients had a secondary site that indicated blood cancer. The longterm results of liver resection of HCC with extrahepatic secondary malignant tumors were similar to those of HCC patients without secondary cancers [26]. Most of these patients succumbed to HCC recurrence. Similar results were reported in a study conducted in southern Japan [27], which found that most cancer recurrences consisted of HCC after liver resection for HCC and extrahepatic secondary malignancies.

Our hematologists routinely monitored patients by performing liver imaging studies during or after treatment for hematologic malignancies. For controlled hematological malignancies, aggressive liver resection for HCC yielded similar early and long-term outcomes as general resectable HCC cases.

The resectability of HCC depends on the patient's general condition and liver function and the tumor location. In the HM group, chemotherapy for hematological malignancy may induce decreased host immunity. Thus, 
the risk of liver resection in these patients may increase. However, the patient selection criteria for HCC in the non-HM group were similar to those applied in the HM group. No operative mortality occurred in the HM group, and the postoperative morbidity was also acceptable. The surgical risks were similar in the HM and non-HM group, particularly in patients whose HCCs were detected during chemotherapy. We believe that if hematological malignancies can be well-controlled, liver resection is still safe in selected patients. HCC resection after prior chemotherapy for hematological malignancy was not a particularly influential factor.

The long-term outcomes of our patients were also affected by the recurrence of $\mathrm{HCC}$, but no recurrence of hematological malignancies was observed; no patient in our series had a recurrent or uncontrolled (progression) hematological malignancy. Therefore, post-operative follow-up of these patients should be especially mindful of the possibility of HCC recurrence, in addition to checking for any evidence of hematological malignancies. If there is suspicion of $\mathrm{HCC}$ recurrence, multidisciplinary therapies should be urgently applied. With respect to the OS in this study, we found that the DFS and OS of HCC patients were similar to those of the general HCC population.

\section{Conclusions}

In conclusion, follow-up after treatment for hematological malignancy should include hepatitis marker, liver functions biomarker, and imaging studies to detect $\mathrm{HCC}$ occurrence especially in patients who are positive for $\mathrm{HBsAg}$ or anti-HCV. Once HCC has been detected, aggressive liver resection is recommended for selected patients.

\section{Abbreviations}

AFP: a-Fetoprotein; Anti-HCV: Anti-hepatitis C antibody; ASA: American Society of Anesthesiology; CT: Computed tomography; DFS: Disease-free survival; HBsAg: Hepatitis B surface antigen; HCC: Hepatocellular carcinoma; HM: Hematologic malignancies; ICG R15: Indocyanine-green 15-min retention rate; IRB: Institutional review board; MRI: Magnetic resonance imaging; OS: Overall survival; RFA: Radiofrequency ablation;

TACE: Transarterial chemoembolization

\section{Acknowledgements}

The authors express their thanks to Mr. JP Chen, a member of biostatistical team of Taichung Veterans General Hospital, for his assistance with the statistical methods.

\section{Funding}

This study was supported in part by a grant from Taichung Veterans General Hospital (No. TCVGH-1064603C).

\section{Availability of data and materials}

Please contact the corresponding author with requests for data.
Ethics approval and consent to participate

This study was approved by the Institutional Review Board of Taichung Veterans General Hospital, Taichung. Research was conducted in accordance with the principals of the 1964 Declaration of Helsinki and its later amendments.

\section{Consent for publication \\ Not applicable.}

\section{Competing interests}

The authors declare that they have no competing interests.

\section{Publisher's Note}

Springer Nature remains neutral with regard to jurisdictional claims in published maps and institutional affiliations.

\section{Author details}

${ }^{1}$ Department of Surgery, Taichung Veterans General Hospital, Section, 4, No. 1650, Taiwan Boulevard, Taichung, Taiwan. ${ }^{2}$ Department of Hematology, Taichung Veterans General Hospital, Taichung, Taiwan. ${ }^{3}$ Department of Anesthesiology, Taichung Veterans General Hospital, Taichung, Taiwan. ${ }^{4}$ Department of Pathology, Taichung Veterans General Hospital, Taichung, Taiwan. ${ }^{5}$ Department of Surgery, School of Medicine, National Yang-Ming University, Taipei, Taiwan. ${ }^{6}$ Department of Surgery, Taipei Medical University, Taipei, Taiwan. ${ }^{7}$ Department of Surgery, Chung-Shan Medical University, Taichung, Taiwan.

Received: 22 June 2017 Accepted: 15 October 2017

Published online: 02 November 2017

\section{References}

1. Greer JP, Arbet DA, Glader B, et al., editors. Wintrobe's clinical hematology. Philadelphia:13th ed. Linppincot: Williams \& Wikkins; 2015.

2. Lan JL, Chen YM, Hsieh TY, et al. Kinetics of viral loads and risk of hepatitis B core antibody-positive rheumatoid arthritis patients undergoing anti-tumor necrosis factor alpha therapy. Ann Rheum Dis. 2011;70:1719-25.

3. Chen YH, Chien RN, Huang YH, et al. Screening and management of hepatitis B infection in rheumatic patients scheduled for biologic therapy: consensus recommendation from the Taiwan Rheumatology Association. Formosan J Rheumatol. 2012;20:1-7.

4. Fried M, Kalra J, llardi CF, et al. Hepatocellular carcinoma in a long-term survivor of acute lymphocytic leukemia. Cancer. 1987;60:2548-52.

5. Grigorian A, O'Brien CB. Hepatotoxicity secondary to chemotherapy. J Clin Transl Hepatol. 2014;2:95-102.

6. Hecht NB, Liem H. Mitochondrial DNA is synthesized during meiosis and spermiogenesis in the mouse. Exp Cell Res. 1986;154:293-8.

7. Huang J, Wang Y, Guo Y, et al. Down-regulated microRNA-152 induces aberrant DNA methylation in hepatitis B virus-related hepatocellular carcinoma by targeting DNA methyltransferase 1. Hepatology. 2010;52:60-70.

8. Cheng AL, Amarapurkar D, Chao Y, et al. Re-evaluating transarterial chemoembolization for the treatment of Hepatocellular carcinoma: consensus recommendations and review by an international expert panel. Liver Int. 2014;34:174-83.

9. Wu CC. Progress of liver resection for hepatocellular carcinoma in Taiwan. Jpn J Clin Oncol. 2017:47:375-80.

10. Sawikko J, Umakuuna M, Makisalo $\mathrm{H}$, et al. Enhanced recovery protocol after liver resection. Br J Surg. 2015;102:1526-32.

11. CC W, Yeh DC, Lin MC, et al. Improving operative safety for cirrhotic liver resection. Br J Surg. 2001;88:210-5.

12. CC W, Peng CM, Cheng SB, et al. The necessity of hepatic vein reconstruction after resection of cranial part of the liver and major hepatic veins in cirrhotic patients. Surgery. 2012;151:223-31.

13. CC W, Cheng SB, Ho WM, et al. Appraisal of concomitant splenectomy in liver resection for hepatocellular carcinoma in cirrhotic patients with hypersplenic thrombocytopenia. Surgery. 2004;136:660-8.

14. Cheng SB, Wu CC, Shu KH, et al. Liver resection for hepatocellular carcinoma in patients with end-stage renal failure. J Surg Oncol. 2001;78:241-7.

15. Liu HT, Cheng SB, CC W, et al. Impact of severe oesophagogastric varices on liver resection for hepatocellular carcinoma in cirrhotic patients. World J Surg. 2015;39:461-8.

16. Pugh RN, Murray-Lyon IM, Dawson IJ, et al. Transection of the oesophagus for bleeding oesophageal varices. Br J Surg. 1973;60:646-9. 
17. Brierley JD, Gospodarowicz MK, Wittekind C. TNM classification of malignant tumors. 8th ed. Oxford: Wiley \& Blackwell; 2017. p. 80-1.

18. Ishak K, Baptista A, Bianchi L, et al. Histological grading and staging of chronic hepatitis. J Hepatol. 1995;22:696-9.

19. Dindo D, Demartines N, Clavien PA. Classification of surgical complications: a new proposal with evaluation in a cohort of 6336 patients and results of a survey. Ann Surg. 2004;240:205-13.

20. Eguchi $\mathrm{Y}$, Hyogo $\mathrm{H}$, Ono $\mathrm{M}$, et al. Prevalence and associated metabolic factors of nonalcoholic fatty liver disease in the general population from 2009 to 2010 in japan: a multicenter large retrospective study. J Gastroenterol. 2012:47:586-95.

21. Billroth $\mathrm{T}$, Von Winiwarter A. Die allgemeine chirurgische Pathologie und Therapie in fünfzig Vorlesungen: Ein Handbuch für Studirende und Aerzte. 10th ed. Edited by Reimer G. Berlin; 1882. p. 1829-894.

22. Warren $\mathrm{S}$, Gates $\mathrm{O}$. Multiple primary malignant tumors: survey of the literature and a statistical study. Am J Cancer. 1932;16:1358-414.

23. Berrington de GA, Curtis RE, Kry SF, et al. Proportion of second cancers attributable to radiotherapy treatment in adults: a cohort study in the US SEER cancer registries. Lancet Oncol. 2011;12:353-60.

24. Koubková L, Hrstka R, Dobes P, et al. Second primary cancers-causes, incidence and the future. Klin Onkol. 2014;27:11-7.

25. Lee JW, Kim JW, Kim NK. Clinical characteristics of colorectal cancer patients with a second primary cancer. Ann Colorproctol. 2014;30:18-22.

26. Xu W, Liao W, Ge P, et al. Multiple primary malignancies in patients with hepatocellular carcinoma: a largest series with 26-year follow-up. Medicine. 2016:95:e3491.

27. Shimada M, Takenaka K, Fujiwara Y, et al. Characteristics of hepatocellular carcinoma associated with extrahepatic primary malignancies in southern Japan. Am J Gastroenterol. 1996;91:754-8.

\section{Submit your next manuscript to BioMed Central and we will help you at every step:}

- We accept pre-submission inquiries

- Our selector tool helps you to find the most relevant journal

- We provide round the clock customer support

- Convenient online submission

- Thorough peer review

- Inclusion in PubMed and all major indexing services

- Maximum visibility for your research

Submit your manuscript at www.biomedcentral.com/submit

) Biomed Central 